NASA Technical Memorandum 105954

ICOMP_92-24; CMOTT-92-12

\title{
Critical Assessment of Reynolds Stress Turbulence Models Using Homogeneous Flows
}

Aamir Shabbir and Tsan-Hsing Shih

Institute for Computational Mechanics in Propulsion

Lewis Research Center.

Cleveland, Ohio

Prepared for the 31st Acrospace Sciences Meeting sponsored by the American Institute of Aeronautics and Astronautics Reno, Nevada, January 11-14, 1993 
.

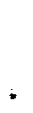




\title{
Critical Assessment of Reynolds Stress Turbulence Models \\ Using Homogeneous Flows
}

\author{
Aamir Shabbir and Tsan-Hsing Shih \\ Institute for Computational Mechanics in Propulsion \\ Center for Modeling of Turbulence and Transition \\ NASA Lewis Research Center, \\ Cleveland, OH 44135
}

\begin{abstract}
In modeling the rapid part of the pressure correlation term in the Reynolds stress transport equations, extensive use has been made of its exact properties which were first suggested by Rotta ${ }^{1}$. These, for example, have been employed in obtaining the widely used LRR model. Some recent proposals have dropped one of these properties to obtain new models. We demonstrate, by computing some simple homogeneous flows, that doing so does not lead to any significant improvements over the LRR model and it is not the right direction in improving the performance of existing models. The reason for this, in our opinion, is that violation of one of the exact properties can not bring in any new physics into the model. We compute thirteen homogeneous flows using LRR (with a recalibrated rapid term constant), IP and SSG models. The flows computed include the flow through axisymmetric contraction; axisymmetric expansion; distortion by plane strain; and homogeneous shear flows with and without rotation. Results show that for most cases a properly calibrated LRR model, which is the most general representation for a model linear in the anisotropic tensor, performs either better or as good as the other two models of the same level.
\end{abstract}




\section{INTRODUCTION}

Modeling of the pressure strain correlation is one of the central issues in the development of Reynolds stress closure models. Most of the popularly used models have utilized the suggestions of Rotta ${ }^{1}$ in developing models for this correlation. From the Poisson equation for the fluctuating pressure he obtained the following expression for the pressure correlation

$$
\begin{aligned}
& \overline{p\left(u_{i, j}+u_{j, i}\right)} / \rho=\frac{1}{4 \pi} \int_{v} \frac{\overline{\left(\overline{\left.\left(u_{p} u_{q}\right)_{q p}\right)^{*}\left(u_{i, j}+u_{j, i}\right)}\right.}}{\left|\mathbf{x}-\mathbf{x}^{*}\right|} d^{3} x^{*} \\
& +\frac{1}{4 \pi} \int_{v}\left(U_{p, q}\right)^{*} \frac{\overline{\left(u_{q, p}\right)^{*}\left(u_{i, j}+u_{j, i}\right)}}{\left|\mathbf{x}-\mathbf{x}^{*}\right|} d^{3} x^{*} \\
& \overline{p\left(u_{i, j}+u_{j, i}\right)}=\overline{p^{S}\left(u_{i, j}+u_{j, i}\right)}+\overline{p^{R}\left(u_{i, j}+u_{j, i}\right)}
\end{aligned}
$$

where the superscript $S$ refers to the so called slow part and the superscript $R$ refers to the rapid part of the pressure correlation ${ }^{2,3,4}$. If we restrict ourselves to the homogeneous turbulence with constant mean gradients (only such flows are considered in this study) then $U_{p, q}$ can be moved outside the integral and one has to model only what else is left inside the integral. For brevity we designate

$$
I_{q i p j}=\frac{1}{4 \pi} \int_{v} \overline{\frac{\left(u_{q}^{*} u_{i}\right), p j}{\left|\mathbf{x}-\mathbf{x}^{*}\right|}} d^{3} x^{*}
$$

Rotta showed that the fourth rank tensor $I_{q i p j}$ has the following properties which are exact.

$$
\begin{aligned}
I_{q i p j} & =I_{i q p j}=I_{q i j p}, \text { symmetry property } \\
I_{q i p i} & =0, \text { incompressibility } \\
I_{q i j j} & =\overline{u_{q} u_{i}}, \text { normalization property }
\end{aligned}
$$

Launder et al. ${ }^{2}$ utilized these constraints in obtaining the so called Launder, Reece and Rodi (LRR) model. They first assumed the most general form of $I_{q i p j}$ which is linear in the anisotropic tensor $b_{i j}=\left(\overline{u_{i} u_{j}} / \overline{q^{2}}-\delta_{i j} / 3\right)$. Then application of the symmetry constraint reduced the number of unknown constants to five. Further application of the incompressibility and normalization constraints reduced the number of unknown constants to one 
which must be determined empirically. Recent study of Speziale et al. ${ }^{5}$ has proposed a model of $I_{q i p j}$ which is linear in the anisotropic tensor $b_{i j}$ and satisfies all of the above constraints except the normalization constraint. This model, therefore, involves four empirical constants. If the normalization constraint is applied to the SSG model then it reduces to the LRR model. So the most general rapid term model which is linear in the anisotropic tensor and satisfies the above mentioned constraints is the LRR model. In our opinion the violation of the normalization property of the rapid term correlation does not bring in any new physics to the model and, therefore, is not the right direction in improving the performance of the currently used models. Direct comparisons of rapid term model with DNS data ${ }^{6}$ showed that the LRR model performs better than SSG model. In this paper it will be further demonstrated, through computations of homogeneous flows, that the properly calibrated LRR model can perform as good as SSG and IP models or even better.

The thirteen homogeneous flows that are numerically computed include flow through axisymmetric contractions and expansions; distortion by plane strain; homogenous shear flows with and without rotation of coordinate axis. In the light of the objective of this study we concentrate only on those models which use a linear (or quasi-linear) model for the rapid term. This, therefore, includes the Launder ,Reece and Rodi ${ }^{2}$ (LRR) model, the isotropization of production (IP) model $^{2}$, and the Speziale, Sarkar and Gatski ${ }^{5}$ (SSG) model. In the next section governing equations and the models tested are given for homogeneous flows. Then a brief description is given of the flows computed followed by the results and conclusions of the study.

\section{GOVERNING EQUATIONS AND MODELS}

For an incompressible homogeneous flow in a rotating frame the Reynolds stress equation can be written as

$$
\begin{aligned}
\frac{D}{D t} \overline{u_{i} u_{j}}= & -\left(\overline{u_{i} u_{k}} U_{j, k}+\overline{u_{j} u_{k}} U_{i, k}\right)-2\left(\overline{u_{i} u_{k}} \epsilon_{m k j} \Omega_{m}+\overline{u_{j} u_{k}} \epsilon_{m k i} \Omega_{m}\right) \\
& +\frac{1}{\rho} \overline{p\left(u_{i, j}+u_{j, i}\right)}-2 \nu \overline{u_{i, k} u_{j, k}}
\end{aligned}
$$


where $\Omega_{m}$ is the rotation rate vector of the coordinates relative to the inertial frame. The model for the pressure strain correlation (denoted by $\Pi_{i j}$ ) and a modeled dissipation rate tensor are needed to complete the second order closure. Usually, the deviatoric part of dissipation tensor is combined with the pressure strain, and a dissipation rate (i.e. $\epsilon$ ) equation is needed.

The form of the dissipation rate equation used by all the models tested here is given by

$$
\frac{D \epsilon}{D t}=-C_{\epsilon 1} \frac{\epsilon}{k} \overline{u_{i} u_{k}} U_{i, k}-C_{\epsilon 2} \frac{\epsilon^{2}}{k}
$$

where $k=\overline{u_{i} u_{i}} / 2 ; C_{\epsilon 1}$ and $C_{\epsilon 2}$ are model coefficients. For LRR and IP models we use $C_{\epsilon 1}=1.44$ and $C_{\epsilon 2}=1.92$ whereas for SSG model $C_{\epsilon 1}=1.44$ and $C_{\epsilon 2}=1.83$.

\section{LRR MODEL}

The LRR model for the pressure correlation term is given by

$$
\begin{aligned}
\Pi_{i j}= & -C_{1} \epsilon b_{i j} \\
& +0.8 k S_{i j}+\frac{\left(18 C_{2}^{\prime}+12\right)}{11} k\left(b_{i k} S_{j k}+b_{j k} S_{i k}-\frac{2}{3} b_{m n} S_{m n} \delta_{i j}\right) \\
& +\frac{\left(20-14 C_{2}^{\prime}\right)}{11} k\left(b_{i k} W_{j k}+b_{j k} W_{i k}\right)
\end{aligned}
$$

where $b_{i j}=\left(\overline{u_{i} u_{j}} / \overline{q^{2}}-\delta_{i j} / 3\right), S_{i j}=\left(U_{i, j}+U_{j, i}\right) / 2$ and $W_{i j}=\left(U_{i, j}-U_{j, i}\right) / 2+\epsilon_{m j i} \Omega_{m}$. The model constants of the LRR model have evolved to slightly different values than those originally recommended by $L R R^{2}$. The value of the Rotta constant $C_{1}$ (in the return to isotropy term) used in the present study is 3.6 This value was used, among others, by Gibson and Launder ${ }^{7}$. The value of the rapid term constant $C_{2}^{\prime}$ (is 0.4 in the original LRR model) used in this study is 0.55 , which is slightly higher than the value of 0.5 recommended by Morris ${ }^{8}$. Therefore, in this paper LRR model will mean equation (5) with $C_{1}=3.6$ and $C_{2}^{\prime}=0.55$. It was found that the value of 0.55 led to improvement in the performance of LRR model for all the flows tested here. (The improvements were slight for the irrotationally strained flows but were significant for the homogeneous shear flows.) 
It should be mentioned that originally LRR obtained the value of this constant from the experiments of Champagne et al. ${ }^{9}$ which were later redone under higher shear rate (see e.g. Tavoularis and Corrsin ${ }^{10}$ ) to obtain more consistent results and these later experiments have been used by several researchers ${ }^{11}$ as a standard benchmark for calibrating model constants. A slightly different value of rapid constant used here is merely a reflection of this fact.

The LRR model is linear in Reynolds stress and satisfies the constraints of incompressibility, normalization and symmetry. It does not satisfy the realizability principle ( which implies that the non-negative quantities such as $\overline{u^{2}}$ should remain non-negative, see Shih and Lumley ${ }^{4}$ for details). It should be further noted that the LRR model is the most general model for the rapid term which is tensorially linear in the anisotropic tensor and satisfies the above mentioned three basic constraints.

\section{IP MODEL}

This model uses the same slow term model as the LRR but for the rapid term keeps only the $C_{2}\left(P_{i j}-2 P \delta_{i j} / 3\right)$ term of the LRR model. When re-written in terms of $b_{i j}, W_{i j}$, and $S_{i j}$ this model is given by the following expression.

$$
\begin{aligned}
\Pi_{i j}= & -C_{1} \epsilon b_{i j}+C_{2} 2 k\left[\left(b_{i k} S_{j k}+b_{j k} S_{i k}-\frac{2}{3} b_{m n} S_{m n} \delta_{i j}\right)\right. \\
& \left.+\left(b_{i k} W_{j k}+b_{j k} W_{i k}\right)+\frac{2}{3} S_{i j}\right]
\end{aligned}
$$

where $C_{1}=3.6$ and $C_{2}=0.6$ were recommended by Gibson and Launder ${ }^{7}$.

\section{SSG MODEL}

The SSG model for the pressure correlation term is given by

$$
\begin{aligned}
\Pi_{i j}= & -\left(C_{1} \epsilon+C_{1}^{*} P\right) b_{i j}+C_{2} \epsilon\left(b_{i k} b_{k j}-\frac{1}{3} b_{m n} b_{m n} \delta_{i j}\right)+\left(C_{3}-C_{3}^{*} I I^{1 / 2}\right) k S_{i j} \\
& +C_{4} k\left(b_{i k} S_{j k}+b_{j k} S_{i k}-\frac{2}{3} b_{m n} S_{m n} \delta_{i j}+C_{5} k\left(b_{i k} W_{j k}+b_{j k} W_{i k}\right)\right.
\end{aligned}
$$

where the definitions of $b_{i j}, S_{i j}$ and $W_{i j}$ are the same as given earlier; $P=-\overline{u_{i} u_{j}} U_{i, j}$ and 
$I I=b_{i j} b_{i j}$. This model involves seven empirical constants and their values are given by

$$
\begin{array}{r}
C_{1}=3.4, C_{1}^{*}=1.80, C_{2}=4.2 \\
C_{3}=\frac{4}{5}, C_{3}^{*}=1.30, C_{4}=1.25, C_{5}=0.40
\end{array}
$$

The rapid part of the SSG model satisfies the constraints of incompressibility and symmetry but it does not satisfy the normalization property. If the constraint of normalization was imposed, the rapid part of SSG model would reduce to LRR model.

\section{FLOWS COMPUTED}

A total of thirteen flows are computed in the present study. These include two axisymmetric contractions (DNS) ${ }^{12}$; two axisymmetric expansions (DNS) ${ }^{12}$; four distortions by plane strain (DNS) ${ }^{12}$; two homogeneous shear flows (one $\mathrm{DNS}^{13}$ and one experiment ${ }^{10}$ ); and three homogeneous shear flows with rotation (LES) ${ }^{14}$. For the flow through the axisymmetric contraction the mean velocity gradient is given by

$$
U_{i, j}=\left(\begin{array}{ccc}
\frac{\partial U}{\partial x} & 0 & 0 \\
0 & -\frac{1}{2} \frac{\partial U}{\partial x} & 0 \\
0 & 0 & -\frac{1}{2} \frac{\partial U}{\partial x}
\end{array}\right)
$$

where $\frac{\partial U}{\partial x}$ is positive. For the flow through axisymmetric expansion the mean velocity gradient tensor is the same as above except that $\frac{\partial U}{\partial x}$ is negative. For distortion by plane strain flow the mean velocity gradient tensor is given by

$$
U_{i, j}=\left(\begin{array}{ccc}
0 & 0 & 0 \\
0 & \frac{\partial V}{\partial y} & 0 \\
0 & 0 & -\frac{\partial V}{\partial y}
\end{array}\right)
$$

where $\frac{\partial V}{\partial y}$ is negative. For the homogeneous shear flow the mean velocity gradient tensor is given by

$$
U_{i, j}=\left(\begin{array}{ccc}
0 & \frac{\partial U}{\partial y} & 0 \\
0 & 0 & 0 \\
0 & 0 & 0
\end{array}\right)
$$

For the homogeneous shear flow in a rotating frame the mean velocity gradient tensor is same as above but in addition it has a non-zero rotation rate which for the cases considered here is given by $\Omega_{i}=(0,0, \Omega)$ where $\Omega$ is constant and represents the rotation rate of the 
non-inertial frame of reference relative to the inertial frame of reference. In all these cases the mean velocity gradients are constant. All these flows constitute an initial value problem and were numerically solved using a fourth order Runge-Kutta finite difference method.

\section{RESULTS}

Figure 1 compares the development of Reynolds stresses computed using these three models in a flow through axisymmetric contraction with the DNS data. (Following Lee et al. ${ }^{12}$ all the stresses will be plotted as a function of the normalized time $S t$.) Here we show a typical case of $S=10.00\left(S k_{o} / \epsilon_{o}=5.573\right.$, case AXL). All the models deviate from the DNS data. However, LRR model gives slightly better results than the SSG model for both the components while IP model performs the worst. Figure 2 shows a comparison for a much higher strain rate case $\left(S=100.00, S k_{o} / \epsilon_{o}=55.73\right.$, case AXM). We note that now all the models deviate even more from the DNS data. We also observe that the LRR model gives better results than the SSG model.

Figures 3 and 4 show a similar comparison for flow through axisymmetric expansion for two different strain rates ${ }^{12}$. For the smaller strain rate flow $\left(S=0.717, S k_{o} / \epsilon_{o}=.408\right.$, case EXO) SSG model reproduces the $\overline{u^{2}}$ development quite well while both IP and LRR models under-predict it. For the $\overline{v^{2}}$ component all the models give similar results. Therefore, for this low strain rate flow SSG model is better than the other two models. For the flow with higher strain rate $\left(S=7.17, S k_{o} / \epsilon_{o}=4.08\right.$, case EXP) the LRR model is in excellent agreement with the DNS data for both the components while both IP and SSG models show over-prediction So for this flow LRR model works the best.

Now we show comparisons for the distortion of turbulence by plane strain for four cases of differing strain rates ${ }^{12}$. We start from the smaller strain rate case. Figure 5 compares the evolution of the three non-zero Reynolds stress components for the flow with strain rate $S=0.65\left(S k_{o} / \epsilon_{o}=0.577\right.$, case PXA). We observe that all the models predict $\overline{u^{2}}$ component reasonably with LRR model slightly better than the other two models. For the $\overline{v^{2}}$ component SSG model is closest to the data where as both LRR and IP models 
slightly under-predict it. For the $\overline{w^{2}}$ component all the three models give about the similar results and predict it reasonably. Figure 6 shows the comparison for the case of $S=1.3$ $\left(S k_{o} / \epsilon_{o}=1.154\right.$, case PXB). The $\overline{u^{2}}$ and $\overline{w^{2}}$ components are reproduced better by the LRR model. However, for the $\overline{v^{2}}$ component SSG model gives better prediction than LRR and IP models. The comparisons for the third case, with strain rate $S=2.6\left(S k_{\circ} / \epsilon_{\circ}=2.309\right.$, case $\mathrm{PXC}$ ), are shown in figure 7 . For $\overline{u^{2}}$ component all the models under-predict the DNS data. LRR model is slightly better than the SSG model. IP model is the worst of the three. For $\overline{v^{2}}$ component IP model works the best. LRR model slightly under-predicts $\overline{v^{2}}$ while SSG over-predicts it. The third component $\overline{w^{2}}$ is over-predicted by all the models with LRR model being better than the other two. Figure 8 shows the similar comparisons for the highest strain rate case $\left(S=25.0, S_{o} k / \epsilon_{o}=22.227\right.$, case PXE). All the three models under-predict the $\overline{u^{2}}$ component. IP model is the worst of the three models. LRR model gives slightly better result than the SSG model for this stress component. For $\overline{v^{2}}$ component LRR model is the best and SSG model is the worst of the three. For the $\overline{w^{2}}$ component all the three models over-predict the DNS data with LRR model being closest to the data. From the above four plane strain flow comparisons, we note that on the overall LRR model works better than the other two models.

Now we turn our attention to homogeneous shear flow. First we show the comparisons with the DNS data ${ }^{13}$ for the shear rate $S=56.56\left(S k_{o} / \epsilon_{o}=2.36\right.$, case C128W). Figure 9 shows that both LRR and SSG models preform equally quite well. On the other hand IP model is off the data for all the components.

Figure 10 shows the same comparison with the homogeneous shear flow experiment of Tavoularis and Corrsin ${ }^{10}\left(S=46.8, S k_{o} / \epsilon_{o}=6.46\right)$. (Following Tavoularis and Corrsin all the Reynolds stresses shown here are normalized by mean velocity along the centerline of their wind tunnel and the streamwise distance is normalized by the height of the wind tunnel.) For the $\overline{u^{2}}$ component LRR model gives the best result whereas SSG and IP models over-predict it. For the $\overline{v^{2}}$ component also the LRR works the best. SSG model slightly over-predicts the data whereas IP model is off by a larger margin. For the $\overline{w^{2}}$ 
component both SSG and IP models reproduce the data very well whereas LRR model over-predicts the data. For the shear stress component LRR performs very well whereas SSG model over-predicts the data and IP model is off the data by a larger margin. So for this experiment LRR model has better overall performance than the other two models.

Finally, we discuss the evolution of $\overline{q^{2}}$ for the case of rotating homogeneous shear flow. Since no experimental or DNS data is available for this flow the comparisons will be made (for two cases) with the LES of Bardina et al. ${ }^{14}$. Bardina ${ }^{15}$ has pointed out that in this case we should be careful in interpreting the comparisons and should use the LES data for judging the model performance in a qualitative fashion. In all the cases shown here the initial conditions corresponded to isotropic turbulence with $\epsilon_{0} / S k_{o}=0.296$. Figure 11 shows the comparisons for the three cases of different Rosby numbers $(=\Omega / S)$. For $\Omega / S=.25$ we note that all the three models significantly under-predict the LES results for $\overline{q^{2}}$; SSG being closest to the LES data and the LRR being the furthest. Qualitatively all the three models reproduce the LES trends. For the case of $\Omega / S=0.50 \mathrm{SSG}$ is in excellent agreement with the LES results. Both IP and LRR give identical results and give a smaller value of $\overline{q^{2}}$ than the LES. For the third case of $\Omega / S=1.0$, all the three models give identical results. Since no LES results are available for this case the only purpose of showing the results is to see how the three models compare with each other.

\section{CONCLUSIONS}

Results were shown from numerical computation of various homogeneous turbulent flows using three different turbulence models. All of these models use a linear (or quasilinear) model for the rapid part of the pressure strain term. The rapid term constant in the LRR model was recalibrated to a slightly different value then the one originally proposed by LRR. Based on their overall performance it is found that the recalibrated LRR model works as good as the SSG and IP models. For the irrotational flows the differences the performance of the LRR model improved over the other two models as the strain rate increased. For the simple homogeneous shear flow experiment LRR model worked better than the SSG model. For the DNS of the same flow both performed equally good. For 
the rotating homogeneous shear flows both SSG and LRR model showed trends similar to those shown by LES with SSG performing closer to LES. It is worth noting that SSG model has seven empirical constants as compared to two in LRR model and still, on the overall, it does not perform better than LRR model. Part of the reason for this may be due to the fact that the SSG model does not satisfy the normalization constraint where as LRR model does. For a model of the rapid pressure strain term which is tensorially linear in the anisotropic tensor and satisfies all the three basic constraints LRR is the most general model. The comparisons shown in this study demonstrate that the model obtained by relaxing normalization property of the pressure correlation term does not lead to any significant improvement over the LRR model and, therefore, this is not the right direction in improving the performance of the current models. 


\section{REFERENCES}

1 Rotta, J.C. "Statistische Theorie Nichthomogener Turbulenz 1. Z. Phys., 1951, 129, 547-572.

${ }^{2}$ Launder, B.E., Reece and W. Rodi. "Progress in the Development of Reynolds-stress Turbulence Closure", J. Fluid Mech., 1975,68, 537-566.

3 Lumley, J.L., "Computational Modeling of Turbulent Flows". Adv. Appl. Mech., 1978, 18, 123-176.

4 Shih, T.-H. and J.L. Lumley, "Modeling of Pressure Correlation Terms in Reynolds Stress and Scalar Flux Equations". Cornell Rep. No. FDA-85-3, 1985, Sibley School of Mech. and Aero. Engg., Cornell University, Ithaca, NY.

${ }^{5}$ Speziale, C.G., S. Sarkar and T.B. Gatski, "Modeling the Pressure-Strain Correlation of Turbulence; An Invariant Dynamical Systems Approach". J. Fluid Mech., 1991, 227, 245-272.

6 Shih, T.-H., and J.L. Lumley, "A Critical Comparison of Second Order Closures with Direct Numerical Simulation of Homogeneous Turbulence", NASA TM 105351, 1992. Fluid Mech., 1976, 8, 183-208.

7 Gibson, and B.E. Launder, "Ground Effects on Pressure in the Atmospheric Boundary Layer". JFM, 1978, 86, 491-511.

8 Morris, P.J., "Modeling the Pressure Redistribution Terms". Phys. Fluids, 1984, $27,7,1620-1623$.

9 Champagne, F.H. , V.G. Harris and S. Corrsin, "Experiments in Nearly Homogeneous Shear Flow", JFM, 1970, 41, 81-139.

10 Tavoularis, S and S. Corrsin, "Experiments in Nearly Homogeneous Turbulent Shear Flow with a Uniform Mean Temperature Gradient", Part 1. 1981, JFM, 104, 311-347.

11 Taulbee, D.B. "Engineering Turbulence Models", Advances in Turbulence, ed. W.K. George and R. Arndt, Hemisphere Publishing Corp.

12 Lee, M. and W.C. Reynolds, "Numerical Experiments on the Structure of Homogeneous Turbulence". Stanford Univ. Report No. TF-24, 1985.

13 Rogers, M, P. Moin and W.C. Reynolds, "The Structure and Modeling of the Hydrodynamic and Passive Scalar Fields in Homogeneous Turbulent Shear Flow", 1986, Stanford Univ. Report No. TF-25.

14 Bardina, J., J.H. Ferziger and W.C. Reynolds, "Improved Turbulence Models Based on Large-Eddy Simulation of Homogeneous, Incompressible Turbulent Flows". Stanford Univ. Report TF-19, 1983.

${ }^{15}$ Bardina, J., (1992) Private communication. 

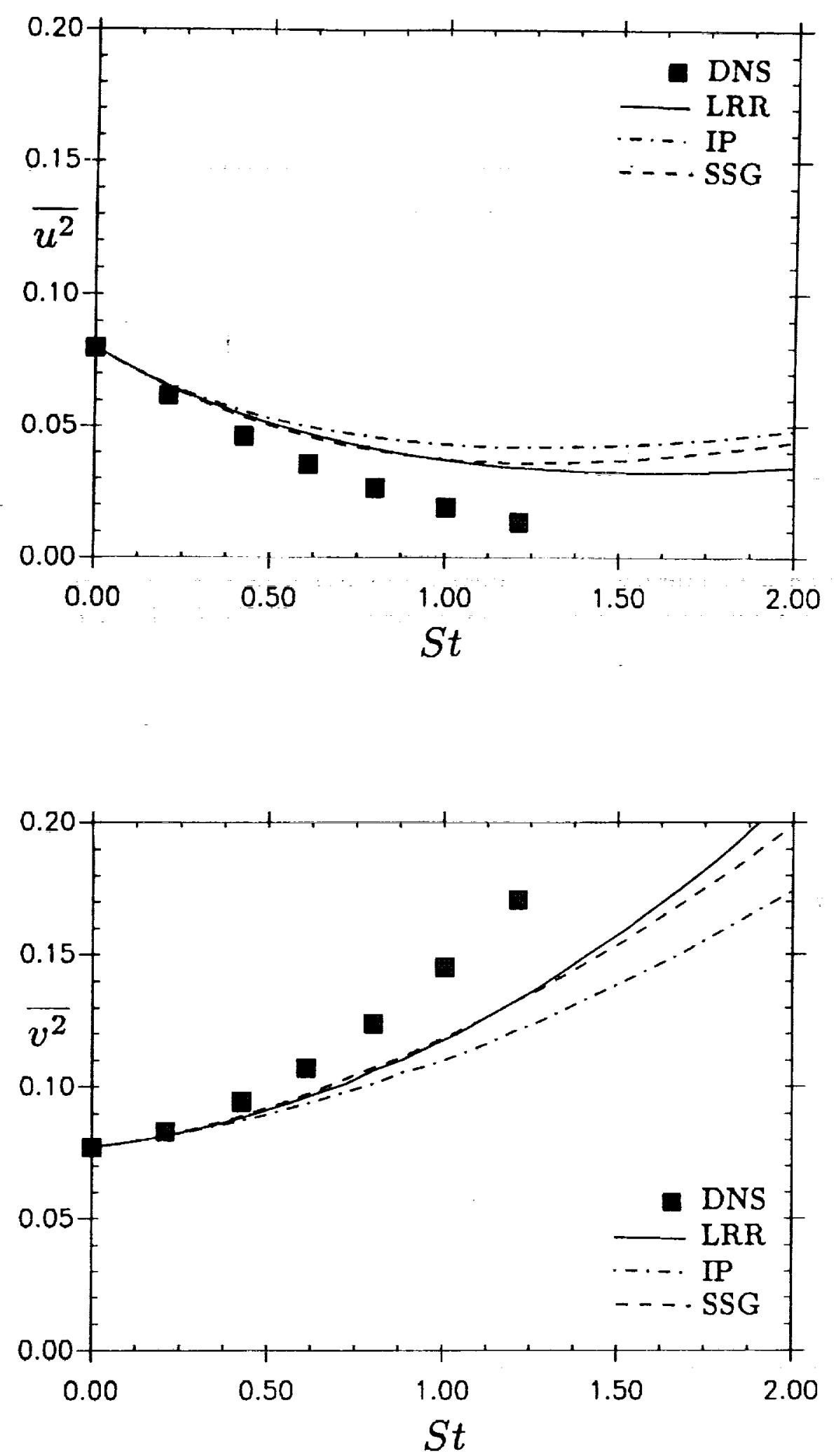

Figure 1. Comparison of the models for flow through axisymmetric contraction with the DNS data of of Lee and Reynolds (1985), case AXL, S=10.0. 

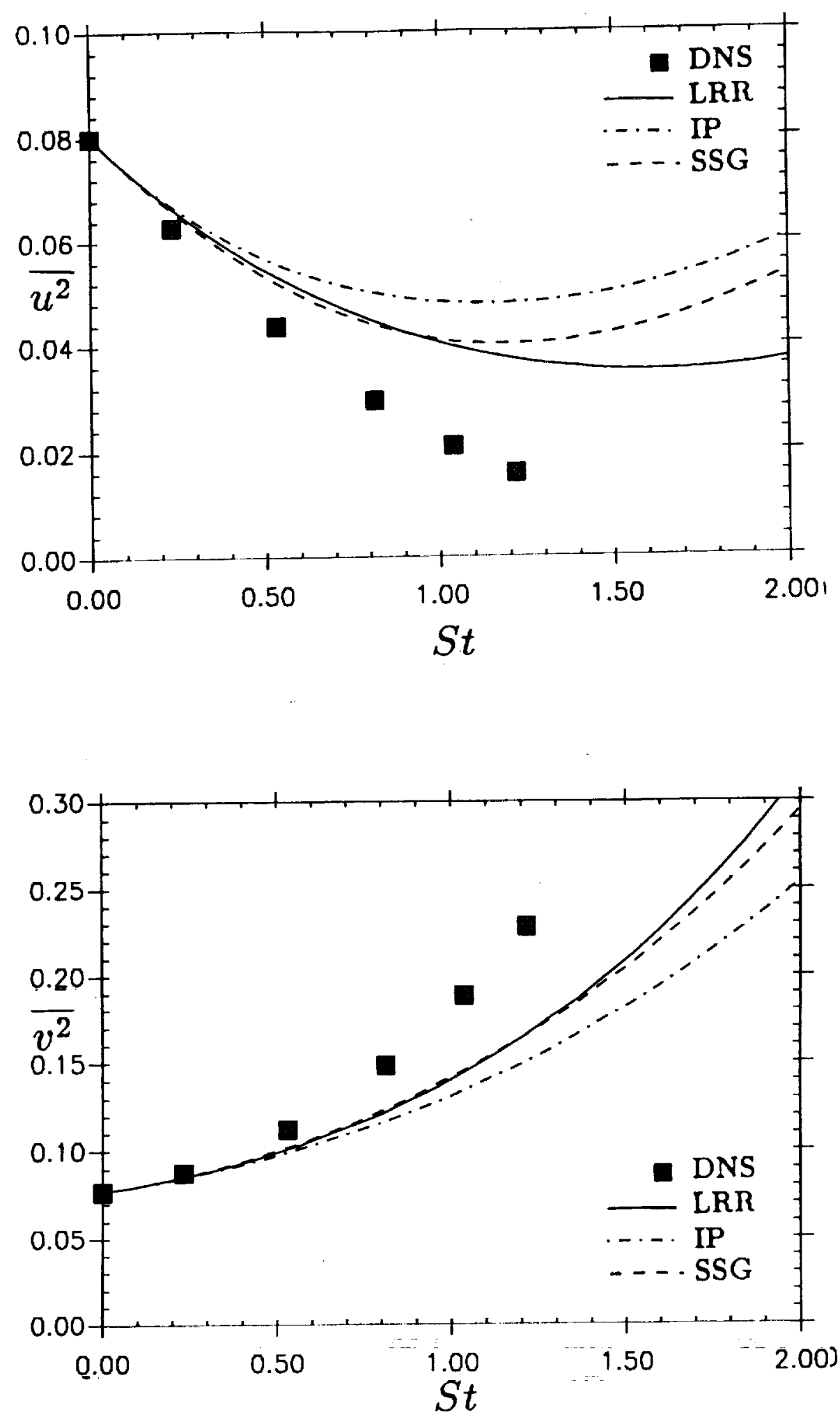

Figure 2. Comparison of the models for flow through axisymmetric contraction with the DNS data of of Lee and Reynolds (1985), case AXM, $S=100.0$. 

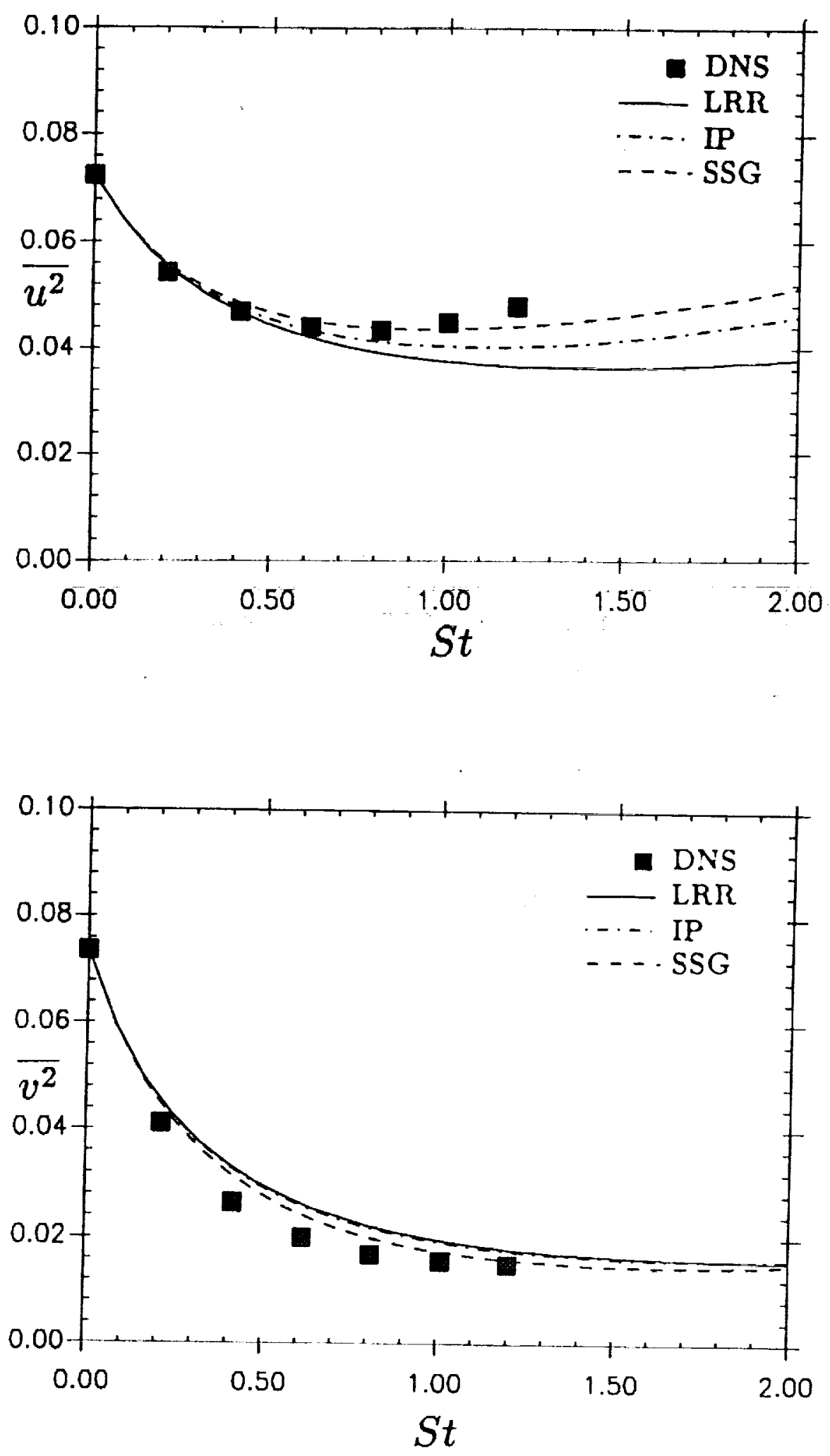

Figure 3. Comparison of the models for flow through axisymmetric expansion with the DNS data of Lee and Reynolds (1985), case EXO, $S=0.717$. 

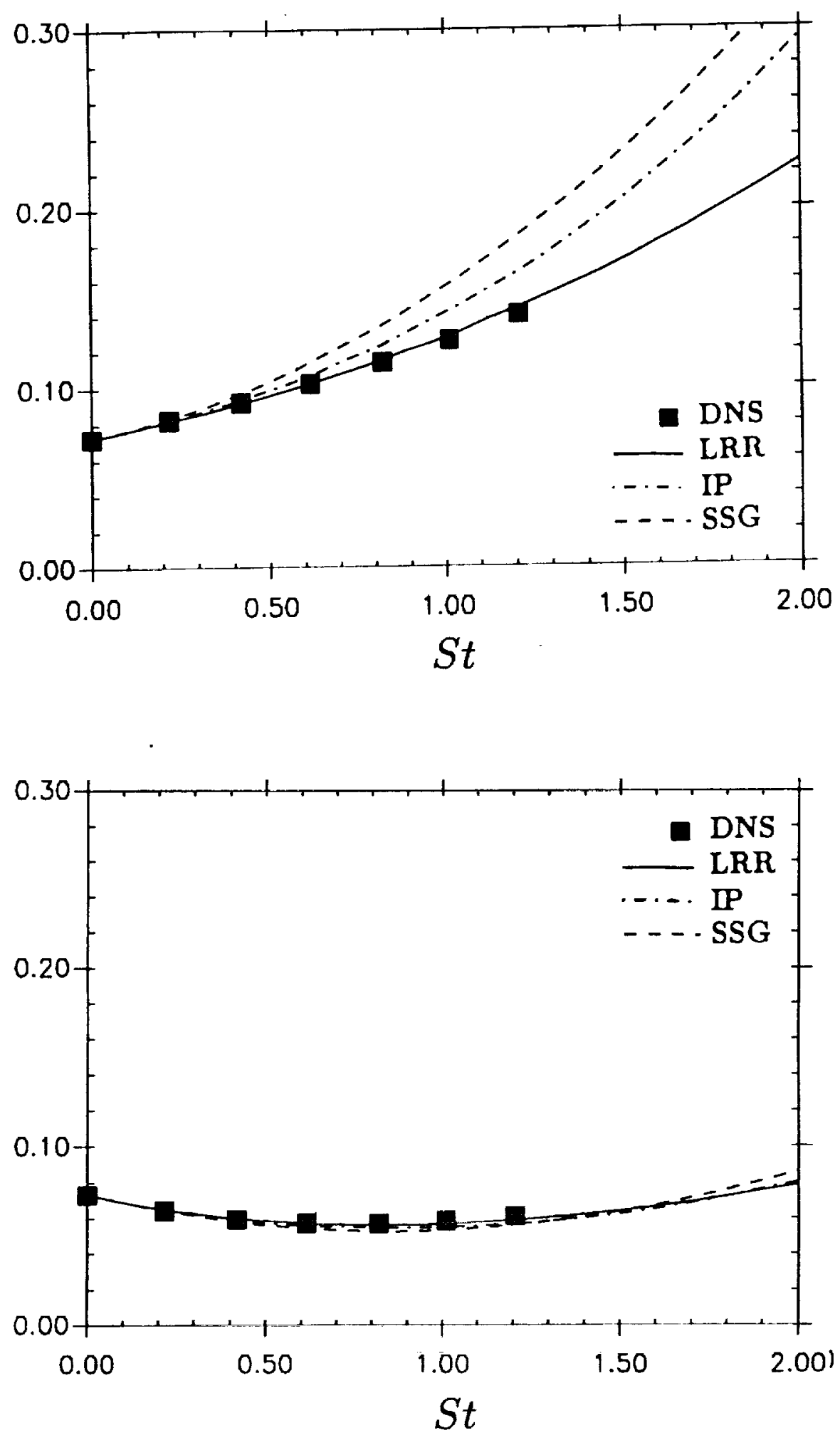

Figure 4. Comparison of the models for flow through axisymmetric expansion with the DNS data of of Lee and Reynolds (1985), case EXP, $S=7.17$. 

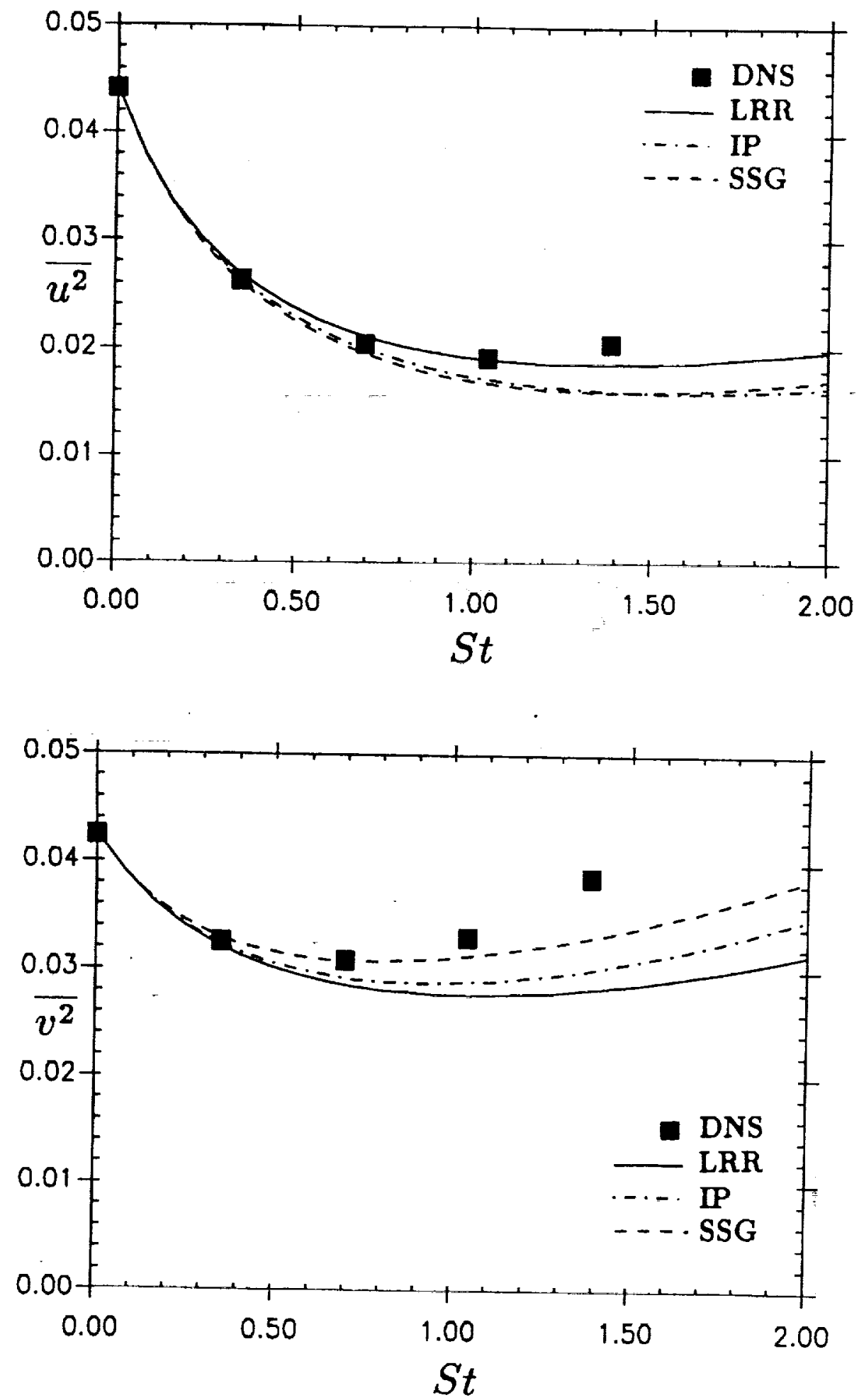

Figure 5. Comparison of the models for distortion by plane strain with the DNS data of Lee and Reynolds (1985), case PXA, $S=0.65$. 


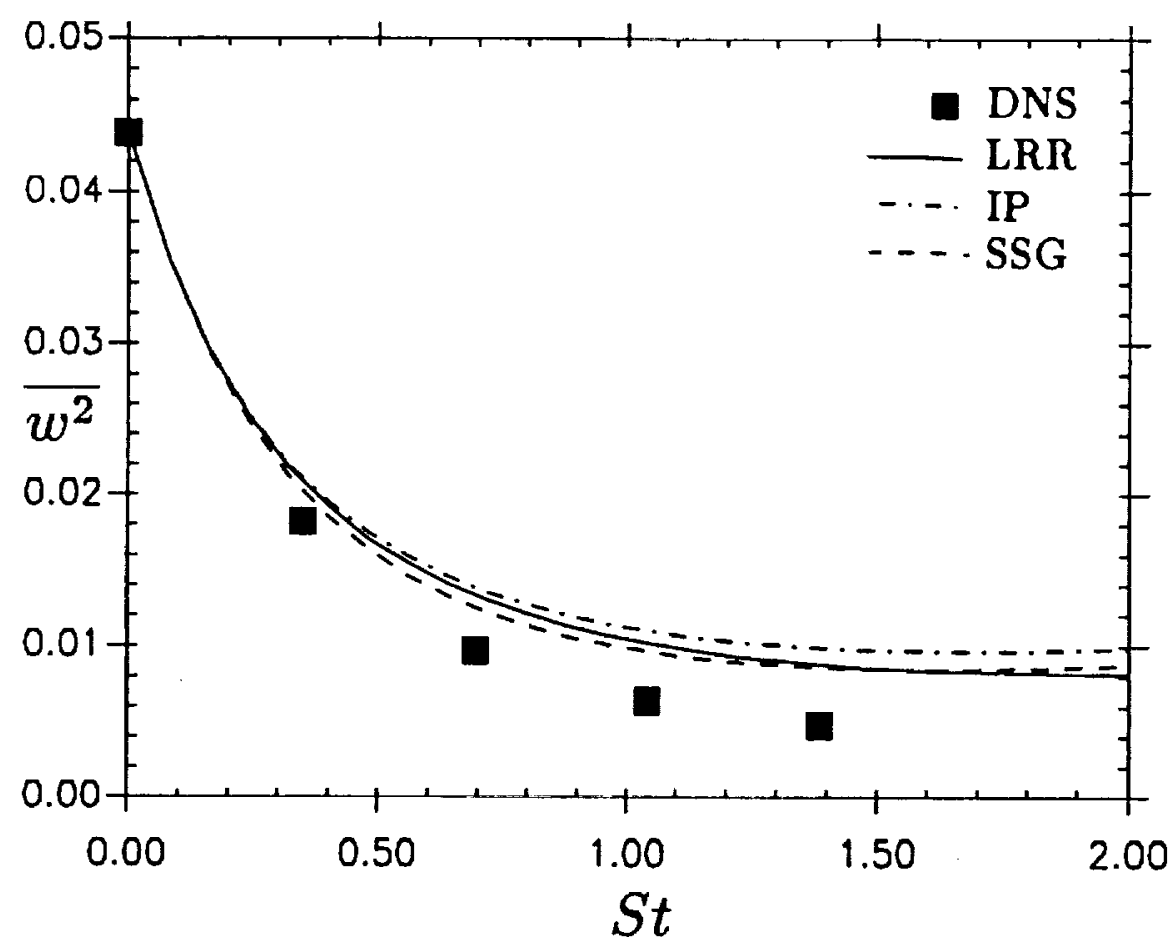

Figure 5 (continued from last page) 

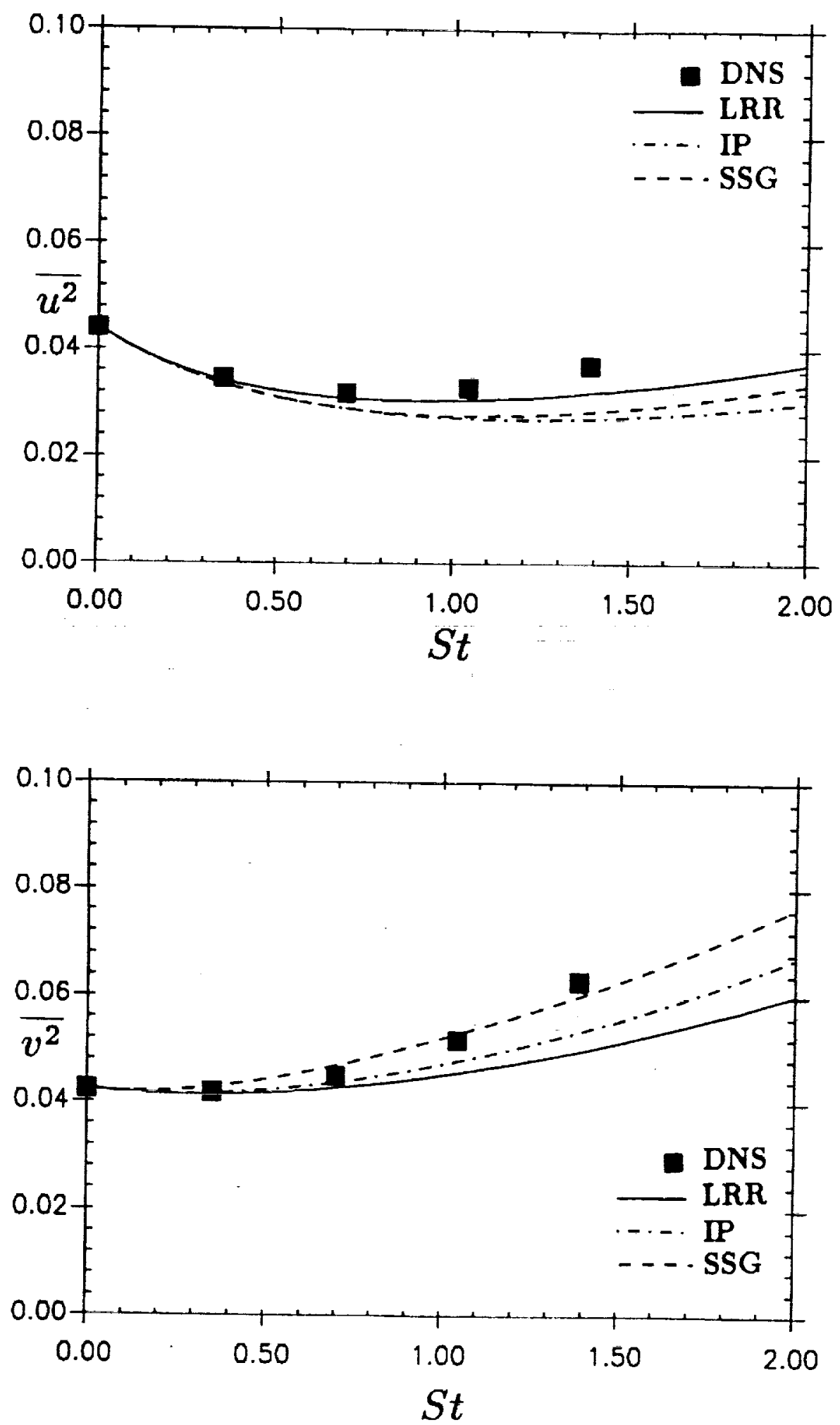

Figure 6. Comparison of the models for distortion by plane strain with the DNS data of Lee and Reynolds (1985), case PXB, $\mathrm{S}=1.3$. 


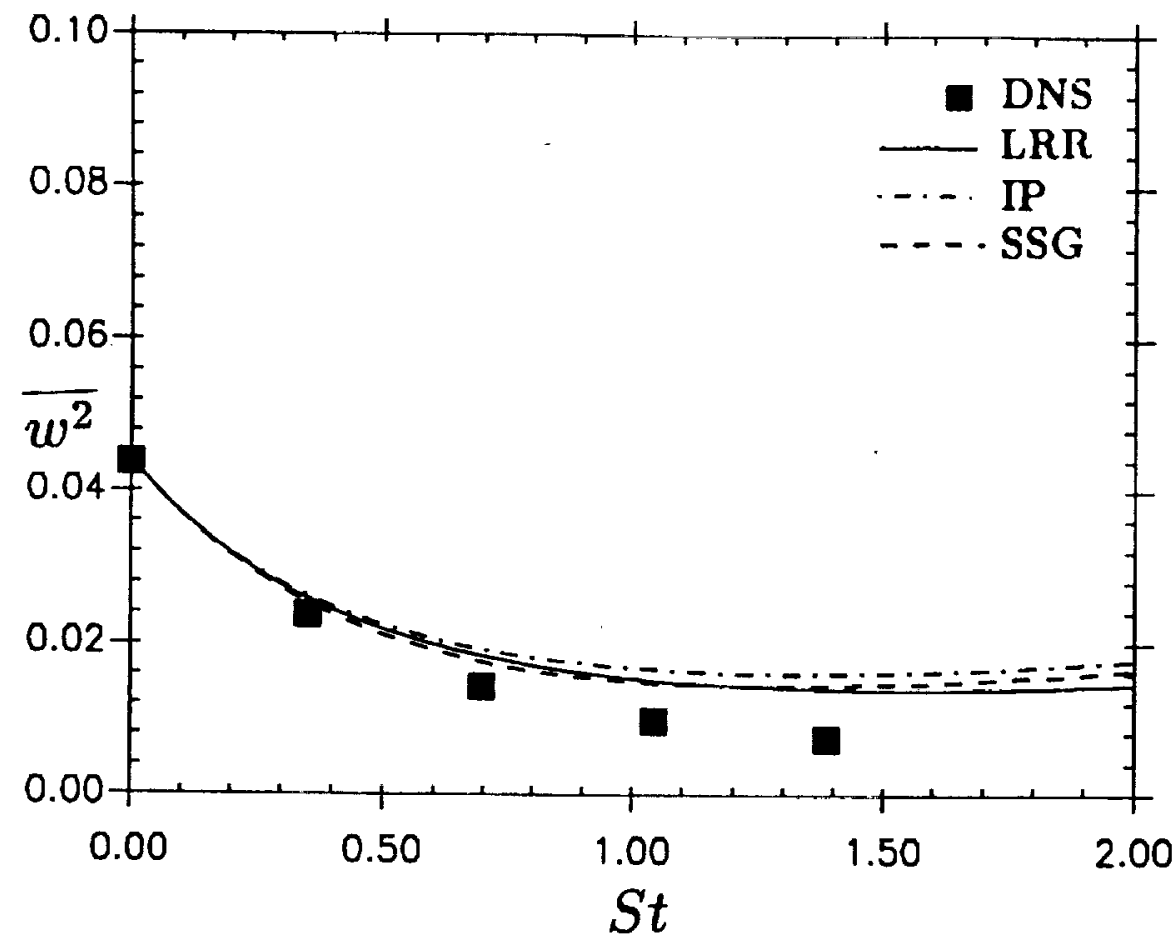

Figure 6 (continued from last page) 

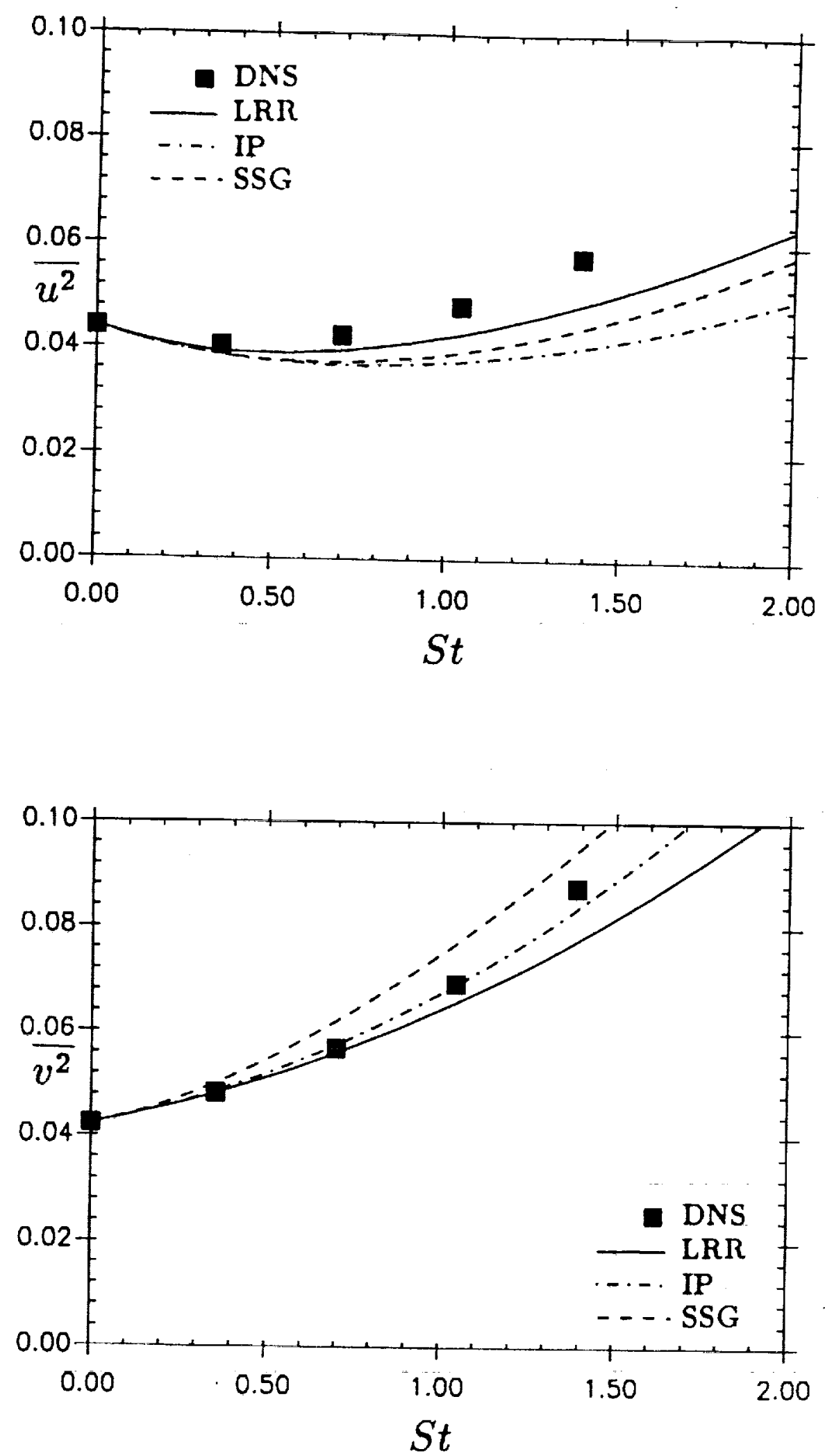

Figure 7. Comparison of the models for distortion by plane strain with the DNS data of Lee and Reynolds (1985), case PXC, $S=2.6$. 


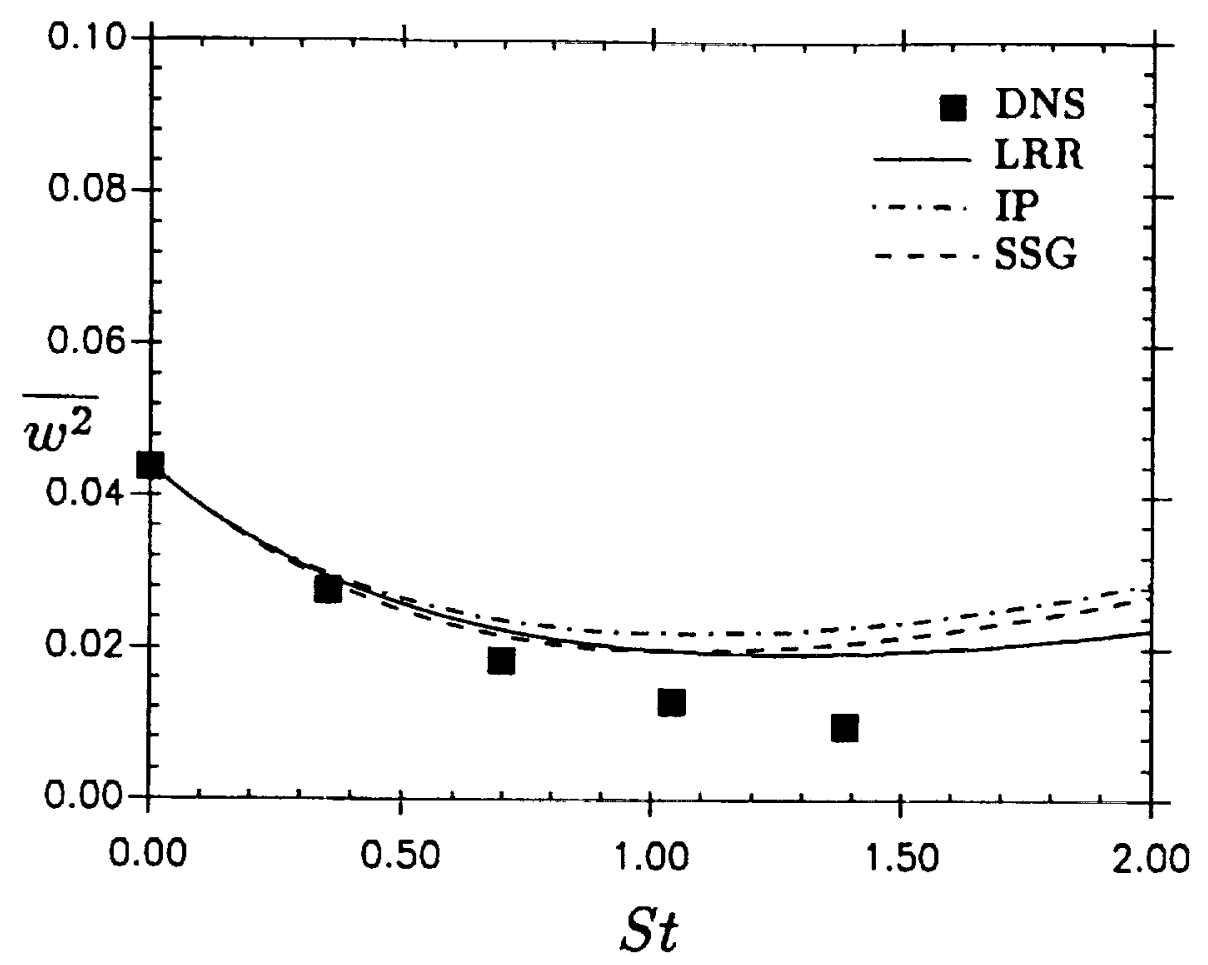

Figure 7 (continued from last page) 

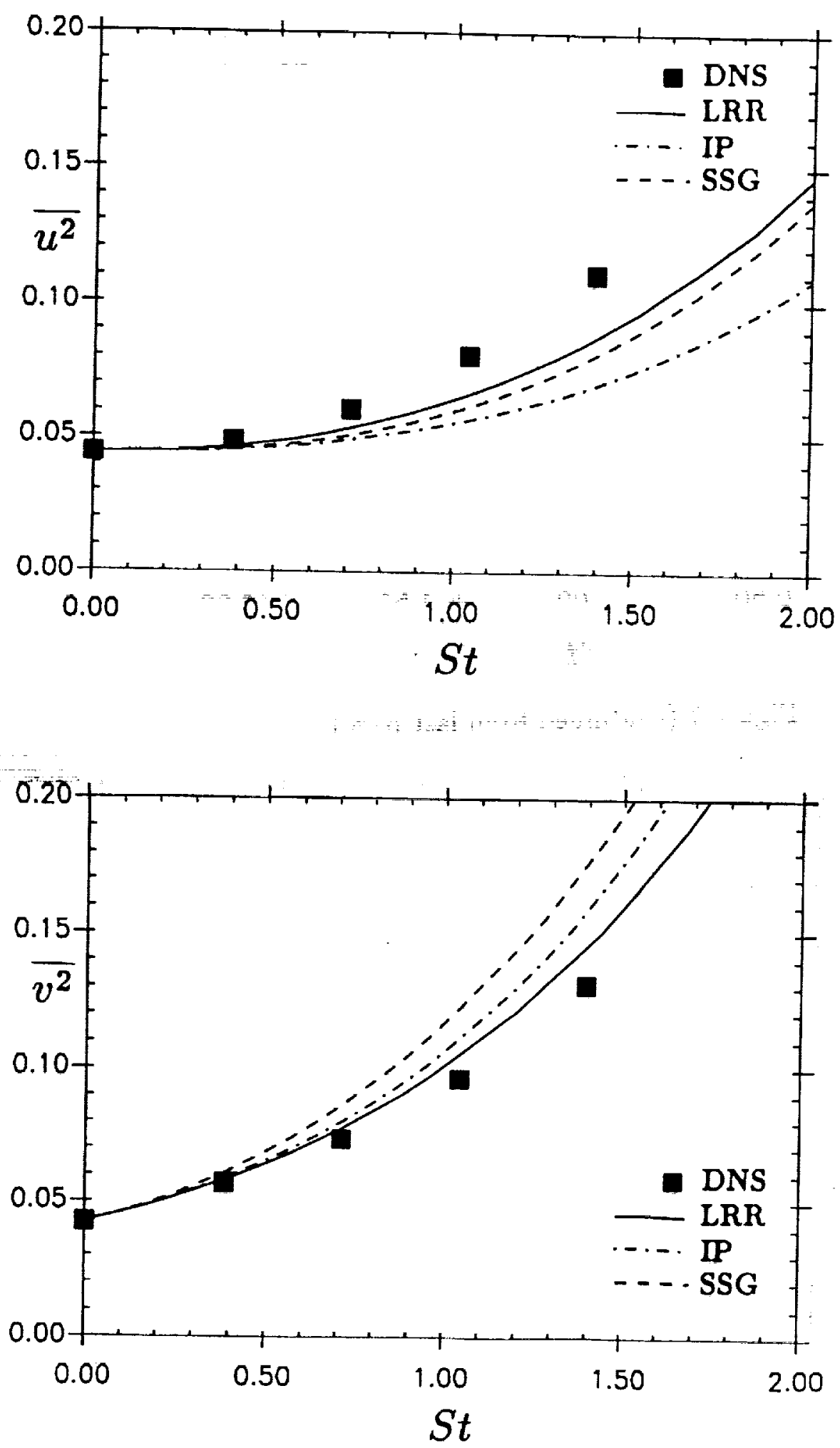

Figure 8. Comparison of the models for distortion by plane strain with the DNS data of Lee and Reynolds (1985), case PXE, $S=25.0$ 


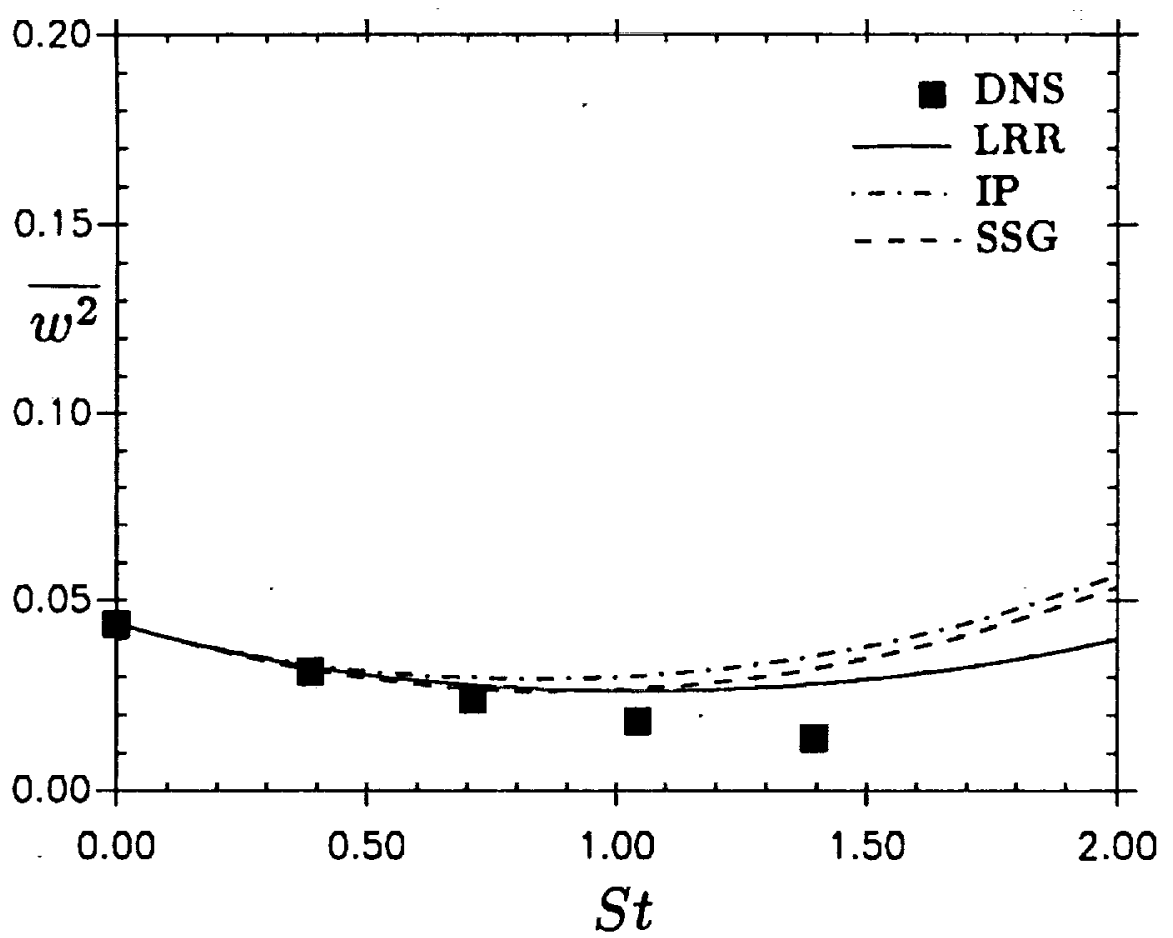

Figure 8 (continued from last page) 

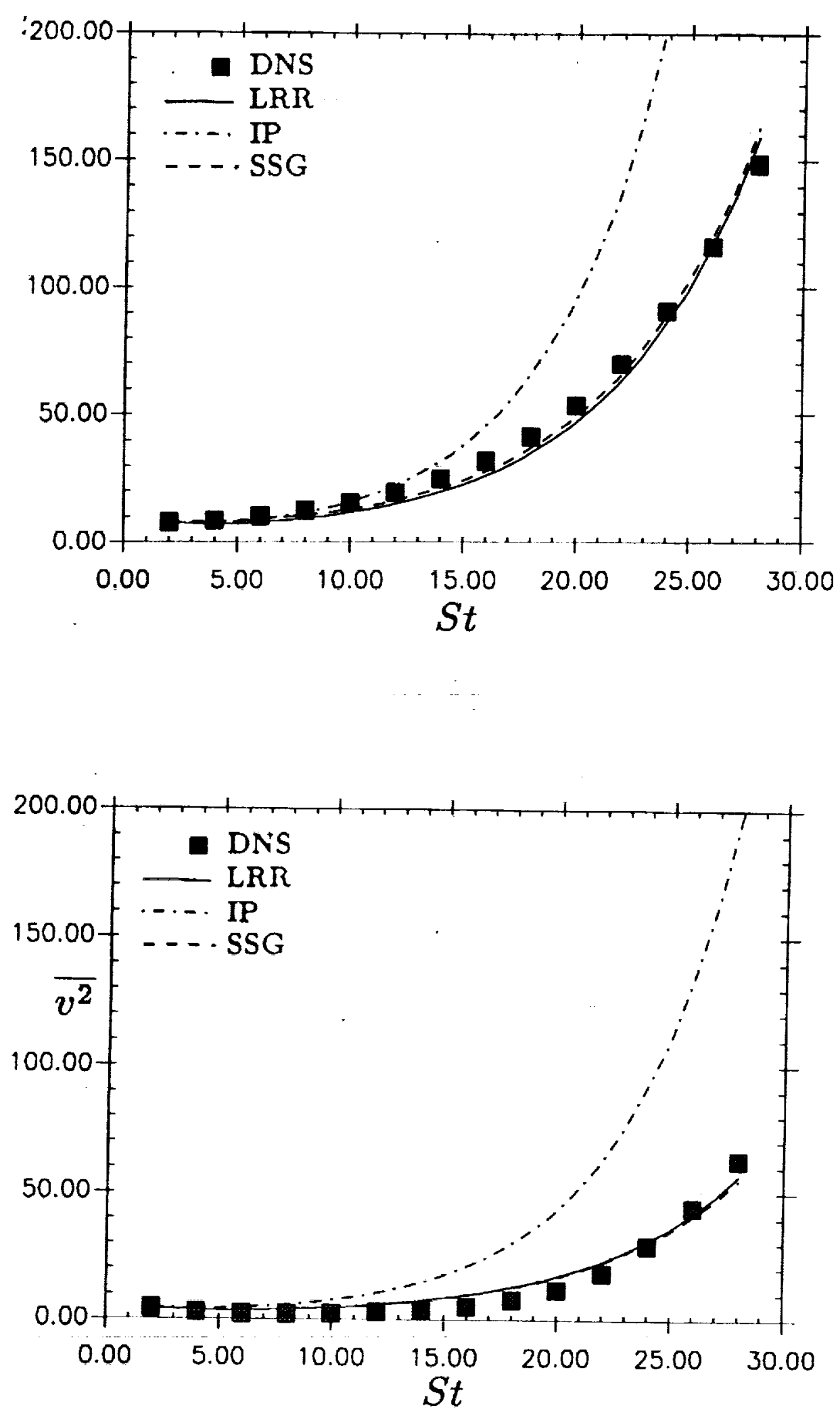

Figure 9. Comparison of the models for homogeneous shear flow with the DNS data of Rogers et al. (1986), case C128W, $S=56.568$. 

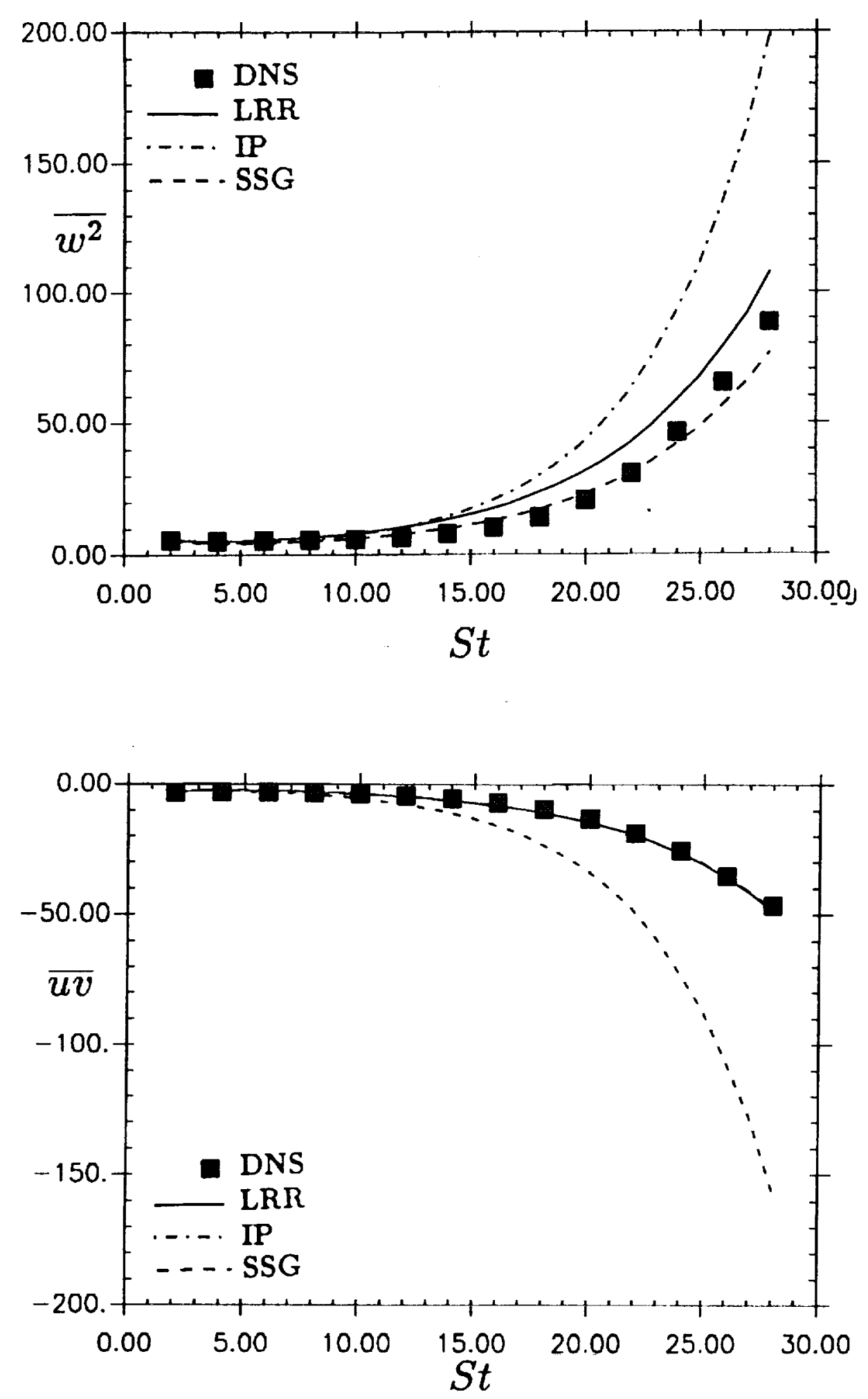

Figure 9 (continued from last page) 

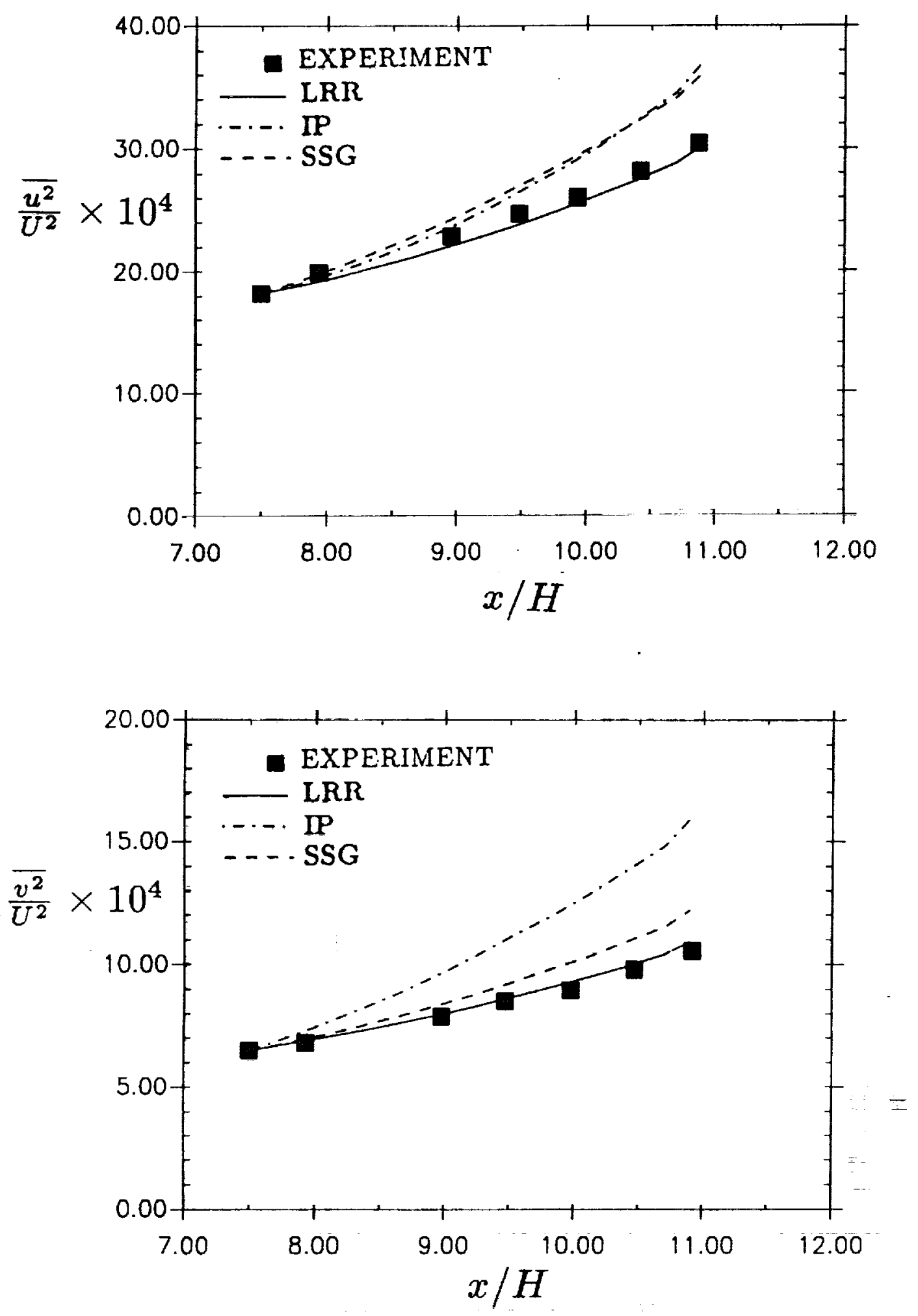

Figure 10. Comparison of the models for homogeneous shear flow with the data Tavoularis and Corrsin (1981). 

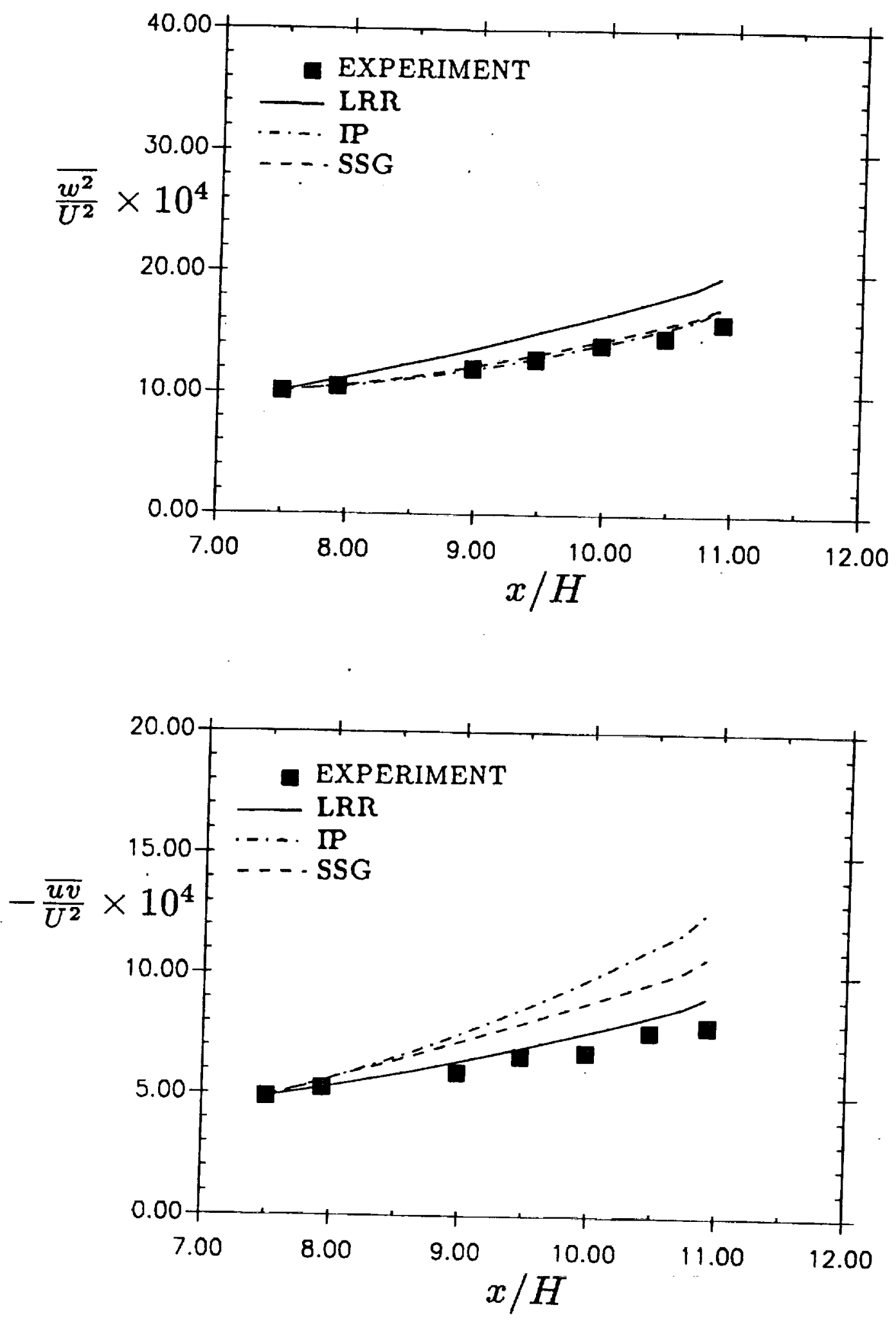

Figure 10 (continued from last page) 

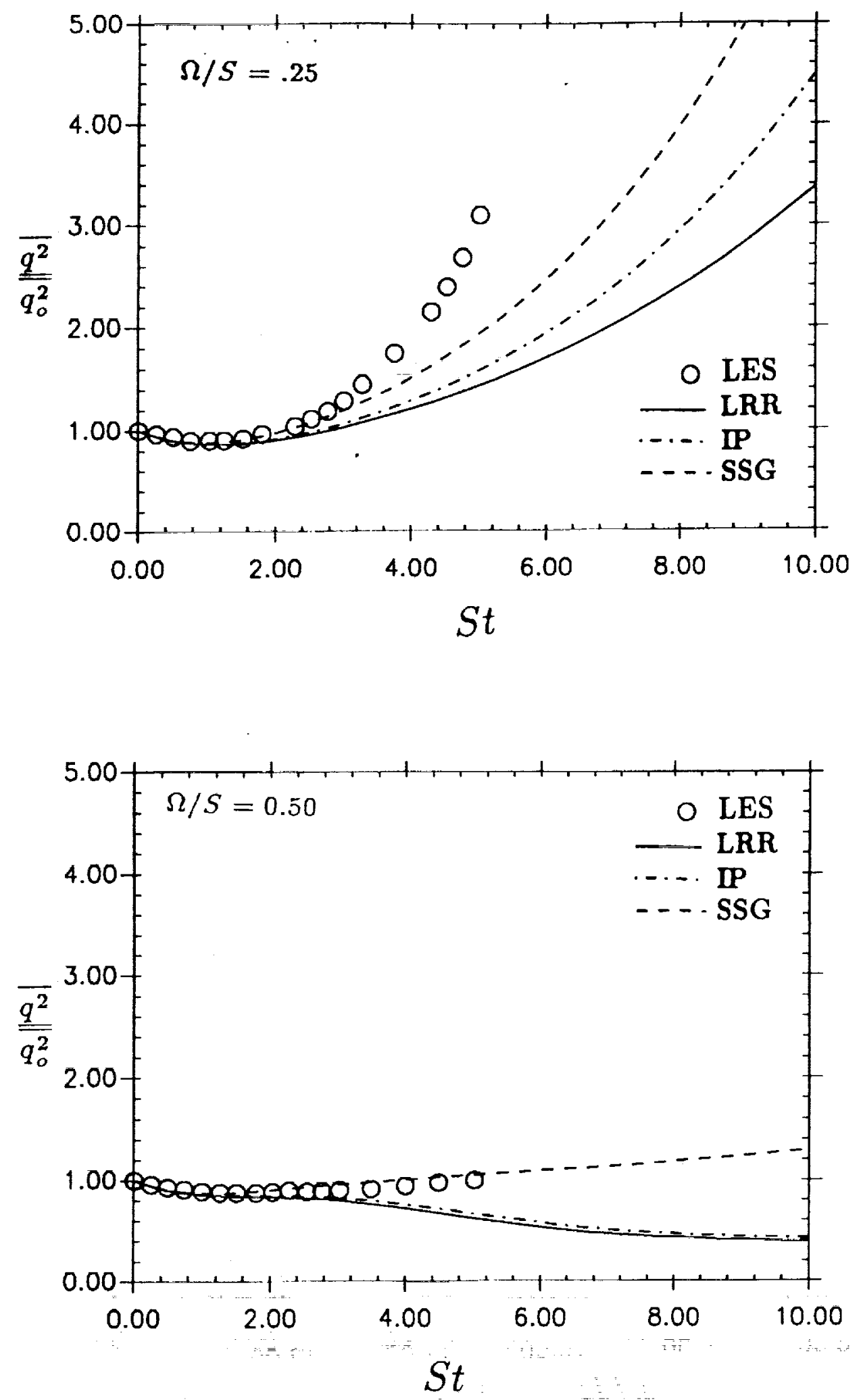

Figure 11. Comparison of the models for the rotating homogeneous shear flow with the large eddy simulation of Bardina $e \bar{t} a \bar{l}$. (1983). 


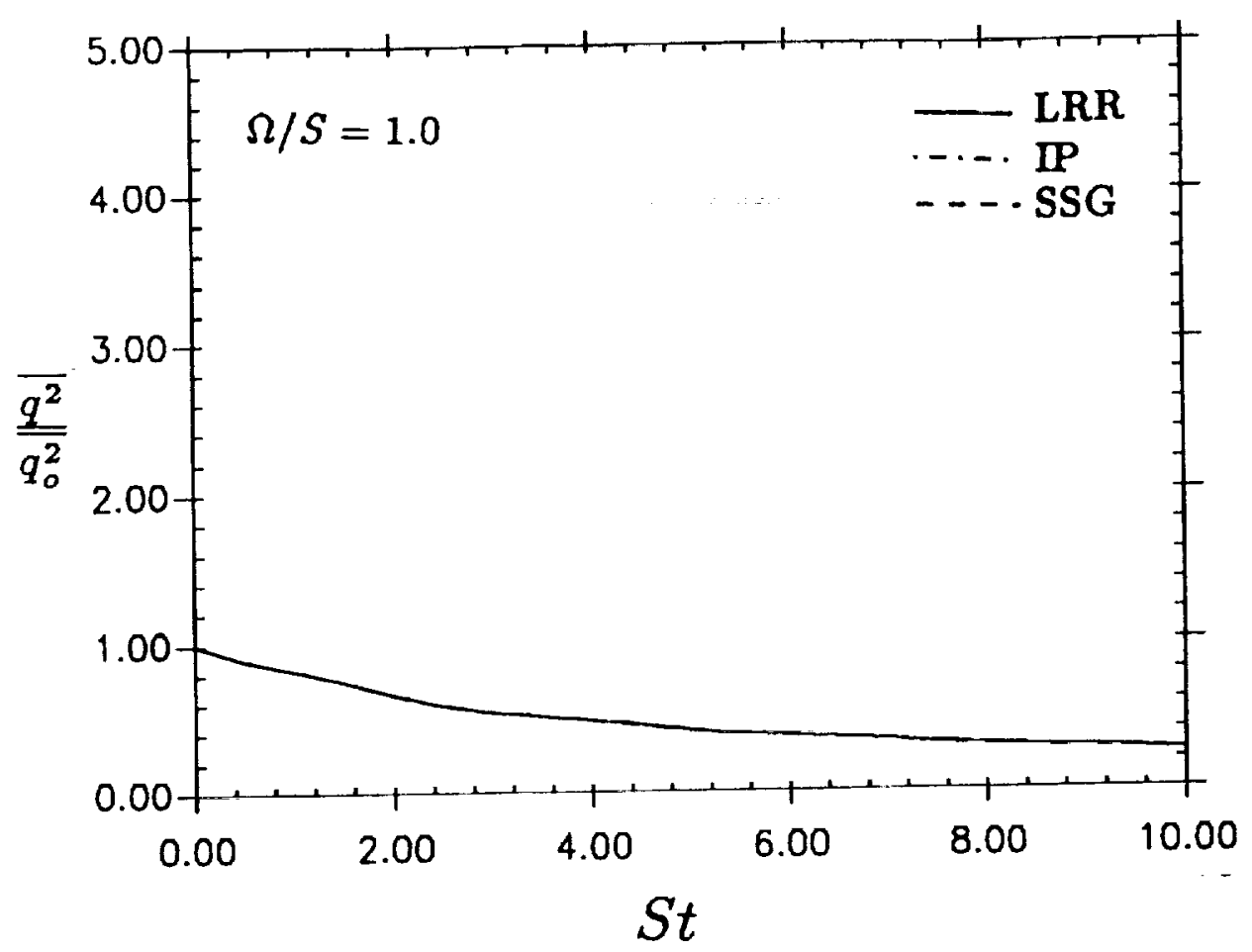

Figure 11 (continued from last page) 


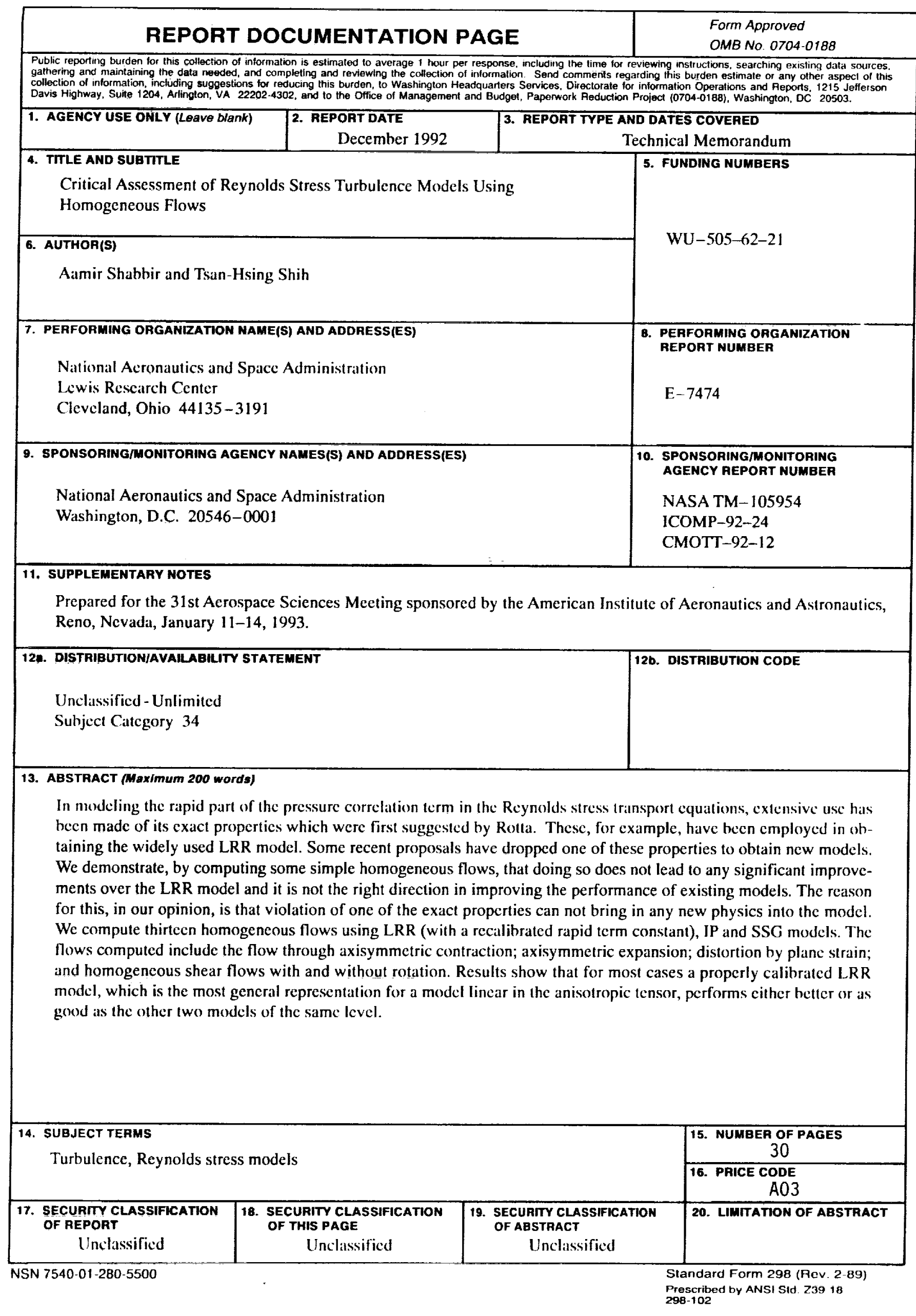

